\title{
Les données marines au service de la gestion des risques littoraux : les réseaux de mesures du Littoral Languedoc- Roussillon
}

\author{
Catherine GARCIA ${ }^{1}$, Cyril VANROYE ${ }^{1}$ \\ ${ }^{1}$ Direction Régionale de l'Equipement du Languedoc-Roussillon \\ Service des Espaces Littoraux, Unité Aménagements et Risques Littoraux \\ 520, allée Henri II de Montmorency, \\ 34064 Montpellier Cedex 2 \\ Tel : 04672051 81-Fax : 0467205084 \\ E-mail : Catherine.Garcia@developpement-durable.gouv.fr \\ E-mail : Cyril.Vanroye@developpement-durable.gouv.fr
}

\section{Résumé :}

Le littoral du Languedoc-Roussillon est fortement concerné par les aléas érosion et submersion marine. L'amélioration des connaissances en matière de dynamique sédimentaire et d'intrusion d'eau de mer nécessite de disposer de données sur les états de mer qui soient fiables, continues et adaptées à la problématique et à la morphologie du Golfe du Lion.

Les réseaux de mesures actuels sur le littoral du Languedoc-Roussillon permettent, d'une part, grâce à des houlographes gérés par la DRE LanguedocRoussillon, l'acquisition permanente de mesures de la houle sur trois sites au large, et d'autre part, grâce à des marégraphes côtiers numériques gérés par le SHOM (Service Hydrographique et Océanographique de la Marine), l' acquisition de la mesure du niveau marin.

Ces données sont également essentielles dans les modélisations hydrodynamiques ( calage des modèles) et permettent d'expliquer, anticiper et comprendre les phénomènes.

\section{Mots-clés :}

Niveaux marins- Hydraulique maritime- mesures- houlographe- marégrapheGolfe du Lion 


\section{$1 \quad$ Introduction}

Le littoral du Languedoc-Roussillon est fortement concerné par les aléas érosion et submersion marine. L'amélioration des connaissances en matière de dynamique sédimentaire et d'intrusion d'eau de mer nécessite de disposer de données sur les états de mer qui soient fiables, continues et adaptées à la problématique et à la morphologie du Golfe du Lion.

Le présent article a pour objet de présenter :

- l'historique des mesures dont dispose à cet effet la DRE LanguedocRoussillon

- les réseaux de mesures actuels et envisagés sur le littoral LanguedocRoussillon qui s'insère dans des réseaux plus globaux gérés par le CETMEF (observatoire CANDHIS) et le SHOM ( réseau RONIM)

- les objectifs de ces mesures

\section{$2 \quad$ Historique des mesures}

Depuis de nombreuses années le SMNLR, puis la DRE Languedoc Roussillon, étudie ces phénomènes d'érosion et de submersion marine et contribue à bâtir et à mettre en oeuvre la politique de gestion des risques littoraux. Ainsi l'acquisition des données marines n'est pas récente.

\subsection{Mesures de niveau d'eau}

La DRE Languedoc-Roussillon dispose de données de niveaux d'eau en mer et dans les étangs du Languedoc-Roussillon sur la période de 1977 à 2001 (soit 88 ans de données cumulées sur l'ensemble des 17 stations concernées) sous forme d'enregistrement papiers qui a nécessité un important travail de numérisation ( 3714 documents papiers) et de calage réalisé par le CETE Méditerranée afin de mieux gérer ces données historiques.

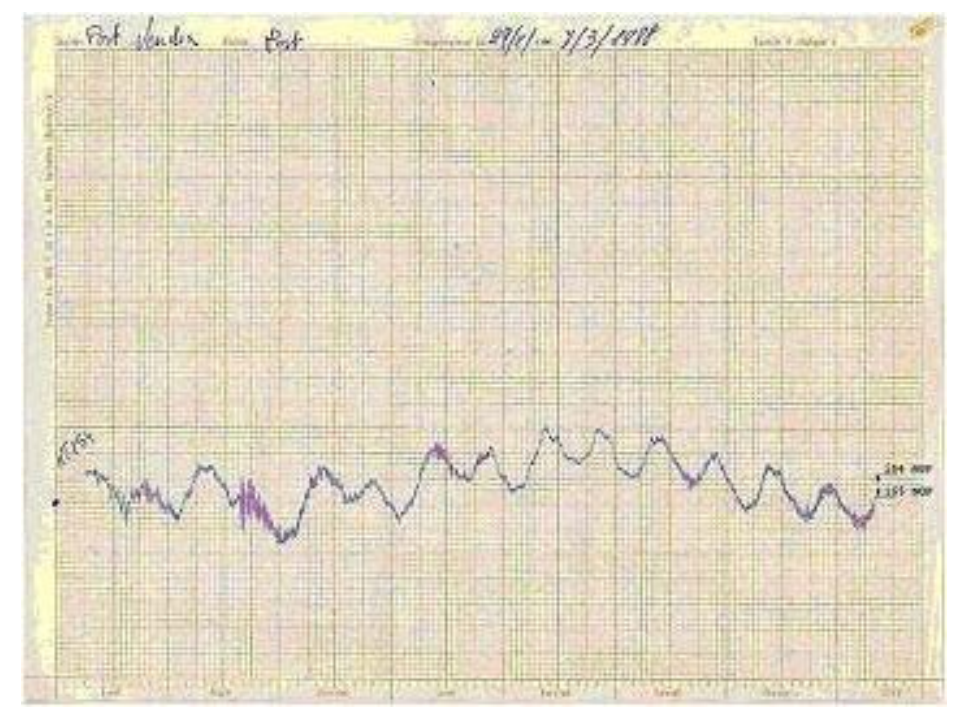

figure 1. Exemple de marégramme papier 
Grâce à cette opération de récupération de niveaux marins la DRE dispose à l'heure actuelle de données numérisées sur les zones géographiques et périodes suivantes :

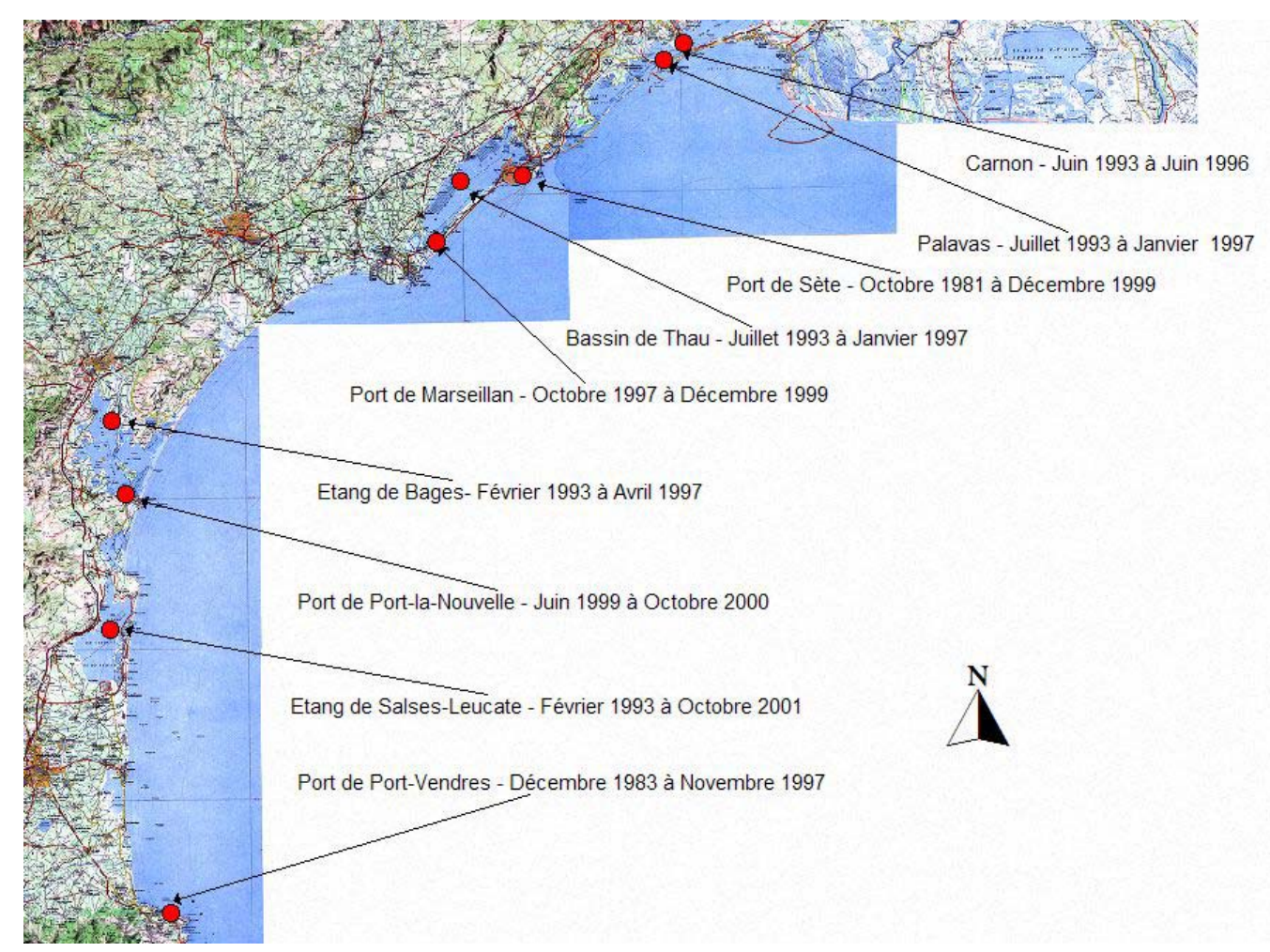

figure 2. Localisation des sites de données de niveau d'eau

\subsection{Mesures de houle}

Les premières données de houle sur le littoral du Languedoc-Roussillon sous format numérique dont dispose la DRE débutent en septembre 1988, date de la mise en place par le SMNLR d'un houlographe omnidirectionnel aux abords de la ville de Marseillan (Département de l'Hérault).

Cependant les mesures de la houle dans le Golfe du Lion sont plus anciennes. En effet, des enregistrements de houle étaient été déjà effectués depuis $1951 \mathrm{au}$ moyen d'un houlomètre comme au Grau de Leucate, depuis 1963 au moyen d'un houlographe à pression comme à Sète et depuis 1970 au moyen d'une bouée Datawell à capteur accélérométrique comme à Sète.

\section{Développements récents de réseaux de mesures}

Afin d'appréhender les phénomènes d'érosion et d'améliorer la gestion des risques il est apparu nécessaire de mettre en place un réseau pérenne de mesures de houle et de niveaux marins en temps réel sur le littoral du LanguedocRoussillon. 
Les réseaux de mesures actuels sur le littoral du Languedoc-Roussillon permettent, d'une part, grâce à des houlographes gérés par la DRE LanguedocRoussillon, l'acquisition permanente de mesures de la houle sur trois sites au large, et d'autre part, grâce à des marégraphes côtiers numériques gérés par le SHOM (Service Hydrographique et Océanographique de la Marine), l'acquisition de la mesure du niveau marin.

\subsection{Réseau de marégraphes : mesures du niveau marin}

Dans le cadre du réseau national RONIM (Réseau d'Observation du Niveau de la Mer) le SHOM (Service Hydrographique et Océanographique de la Marine) a mis en place en octobre 2007 deux MCN (Marégraphes Côtiers Numériques) en remplacement des installations marégraphiques existantes :

- à Sète (département de l'Hérault), en partenariat avec la Région LanguedocRoussillon

- à Port-Vendres (département des Pyrénées-Orientales), en partenariat avec le Conseil Général des Pyrénées-Orientales et la DRE Languedoc-Roussillon
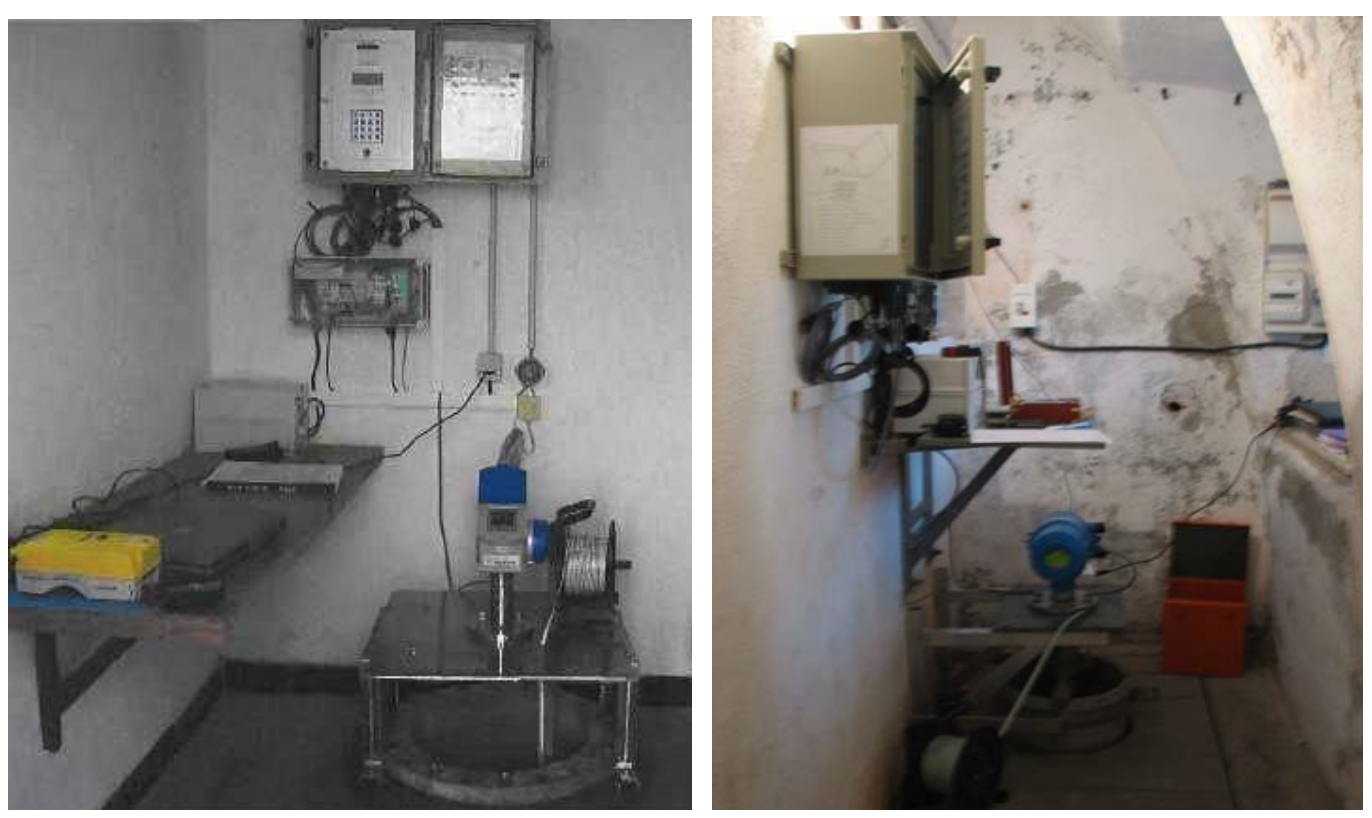

Figure 3. MCN Site de Sète

Figure 4. MCN Site de Port-Vendres

Les MCN sont constitués d'un capteur de distance radar pour une mesure précise de la hauteur d'eau (une mesure par seconde) et d'une centrale d'acquisition programmée pour acquérir toutes les 10 minutes une mesure moyennée sur une durée d'intégration de 2 minutes. La moyenne des mesures sur 
2 minutes permet de filtrer de façon numérique les mouvements verticaux à haute fréquence (houle, clapot).

Les mesures de hauteurs d'eau sont associées à des capteurs annexes :

- un capteur de pression atmosphérique qui fournit une mesure horaire de pression atmosphérique

- un GPS permanent à Sète pour l'étude de la stabilité du site, utile dans le cadre de la problématique de l'élévation du niveau de la mer

Les données temps différé du réseau RONIM sont collectées au SHOM en interrogeant directement les MCN par une liaison modem. Les données sont ensuite traitées, validées et archivées au SHOM dans la base de données nationale de hauteurs d'eau.

Les données des MCN sont mises à disposition en temps différé et gratuitement pour l'enseignement et la recherche sur le site FTP du portail SONEL (www.sonel.org). Elles sont diffusées par le bureau prestations du SHOM pour les autres applications (bp@shom.fr $)$.

Les données temps différé des marégraphes RONIM ont de nombreux champs d'application dont voici une liste non-exhaustive :

- la réduction des sondages bathymétriques ;

- la mesure des surcotes et décotes (ondes de tempêtes, tsunamis)

- la définition des références verticales maritimes ;

- la calibration des radars altimètres Topex-Poséïdon, ERS et Jason ;

- la réalisation de modèles océaniques et d'hydrodynamique côtière.

- la détermination des constantes harmoniques nécessaires à la prédiction de la marée ;

- l'étude de l'évolution du niveau moyen de la mer;

- l'étude statistique des surcotes et décotes, des niveaux extrêmes ;

Ces applications nécessitent des mesures suffisamment précises, et des données correctement référencées (rattachées à des repères terrestres de nivellement pour la conservation des niveaux de référence). Les trois dernières applications citées nécessitent de plus des observations de longue durée.

Les centrales d'acquisition des MCN RONIM peuvent aussi recevoir des systèmes de transmission temps réel de données (Internet, satellite) et fournir des données à haute fréquence. Cette capacité temps réel est actuellement développée au SHOM avec le soutien des partenaires locaux du réseau et est indispensable à la mise en place de systèmes d'alerte aux submersions marines (ondes de tempêtes ou tsunamis - projet SATANEM-) et au développement de l'océanographie côtière opérationnelle. 


\subsection{Réseau de houlographes : mesure de la houle}

La DRE Languedoc-Roussillon dispose à l'heure actuelle d'un réseau de houlographes constitué de trois bouées directionnelles de mesure de la houle (Datawell MKIII ) mises en service en 2006 au large de Sète (département de l'Hérault) et Leucate (département de l'Aude) et fin 2007 au large de Banyuls /mer (département des Pyrénées-Orientales).
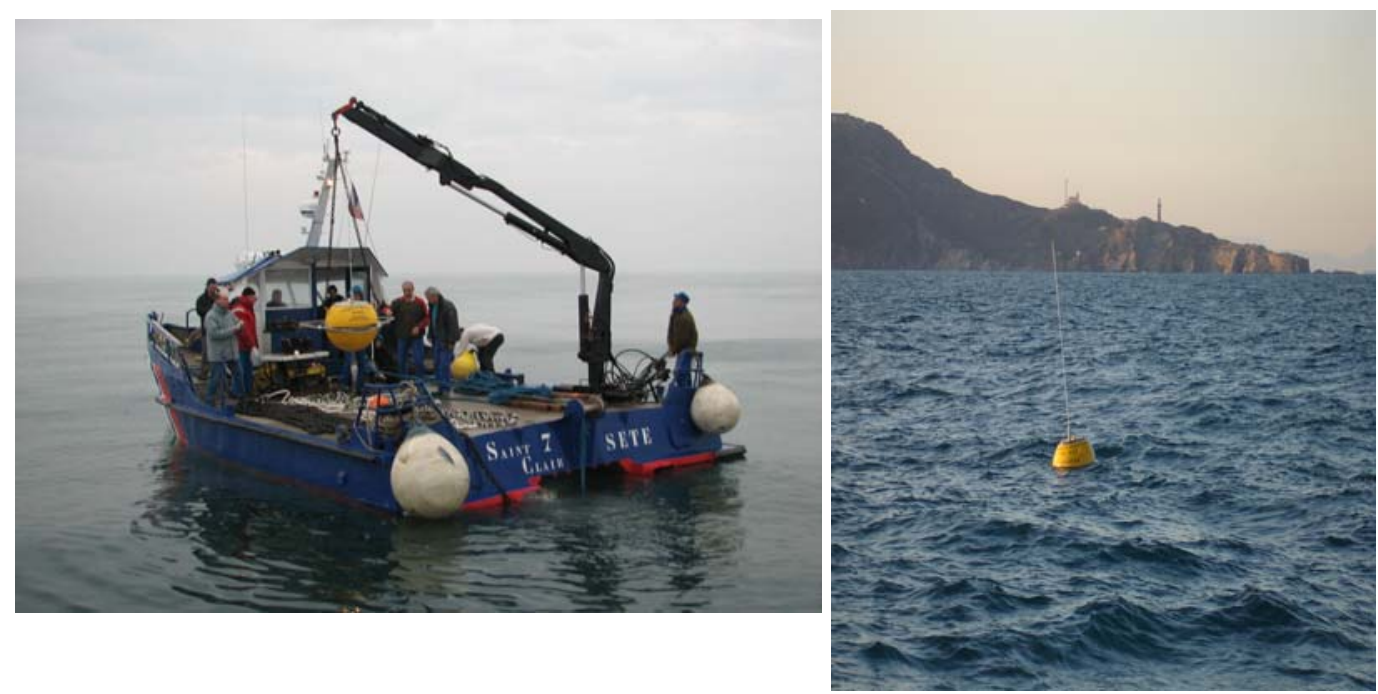

Figure 5 . Opération de mouillage d'un houlographe avec la subdivision des Phares et Balises de Sète

Figure 6. Bouée Datawell au large de Banyuls/Mer

Principe de la mesure de houle : la bouée de houle enregistre en continu la hauteur des vagues, leur direction et leur période. Toutes les 30 minutes sont récupérées après traitement les données brutes suivantes :

- la hauteur significatives (Hs) ou $(\mathrm{H} 1 / 3)$ et la période significative (Ts) ou (TH1/3)

- la hauteur moyenne (Hmoy) et la période moyenne (Tmoy)

- la hauteur maximum (Hmax) et le période maximum (Tmax) ou (THmax)

Comme présenté dans la figure 7 ci-après, le fonctionnement du réseau de mesure de houle se présente comme suit :

- la D.R.E. collecte en temps réel les données brutes (non validées) des houlographes, alimente la base de données SOLLAR ( Système d'Observation du 
Littoral Languedoc-Roussillon) sur le site intranet de la DRE LanguedocRoussillon et les stocke sur un site FTP ( File Transfer Protocol).

- le C.E.T.M.E.F (Centre d'Etudes Technqiues Maritimes et Fluviales) alimente en temps réel sur leur site internet la base de données CANDHIS (Centre d'Archivage National de Données de Houles In-Situ) via le serveur FTP de la D.R.E.

- les données brutes transmises par la D.R.E. sont traitées et validées par le CETMEF et sont ensuite archivées dans la base de données nationale CANDHIS et dans la base régionale SOLLAR.

Le C.E.T.M.E.F est seul chargé de valider définitivement les données. Cependant, dans le cadre de la gestion de crise et pour fournir des éléments en vue de l'établissement des rapports de catastrophes naturelles (CAT-NAT), la D.R.E. a besoin de disposer très rapidement d'une première validation sommaire des données. Pour cela le C.E.T.M.E.F. met à la disposition de la D.R.E. son outil de validation nommé « Houle 4 ». Les données ainsi validées sommairement par la D.R.E. pour la rédaction du rapport ne seront versées dans les bases de données CANDHIS et SOLLAR qu'après avoir été complètement validées par le C.E.T.M.E.F. dans le cadre de la procédure classique.

Principe de fonctionnement de la chaîne de traitements des données de houle :

- le houlographe transmet par radio ses trois déplacements (roulis, tangage et pilonnement) avec une fréquence d'échantillonnage de $1.28 \mathrm{~Hz}$.

- le logiciel "VagueDir" reçoit ces informations, les stocke et effectue un premier traitement afin d'obtenir les valeurs caractéristiques qui seront exploitées en temps réel.

- ces données brutes sont collectées par la DRE qui les transmet ensuite au CETMEF pour validation. L'outil de validation du CETMEF, le logiciel nommé « Houle4 » permet de réaliser les traitements suivants:

-analyse vague par vague de type "Down Zéro Crossing"

-analyse vague par vague de type "Up Zéro Crossing"

-analyse spectral

Une quarantaine de paramètres sont ainsi calculés. Afin que ces paramètres soient validés, différents test sont effectués:

- test d'accélération,

- test du plateau (mesures ne passant pas par le zéro durant une trop

longue durée),

- test du zéro électrique prolongé (mesures dont l'amplitude reste proche de zéro durant une trop longue durée)

A l'issue de ces traitements, «Houle 4 » vérifie la cohérence des résultats (HMax par rapport au H1/3, périodes anormalement longues).De plus, « Houle 
Instrumentation, mesures, imagerie et télédétection

4 » permet l'édition de graphes d'évolution mensuelle de certains des paramètres calculés.

Les données sont mises à disposition gratuitement en :

- en temps réel, sur le site intranet de la DRE Languedoc-Roussillon et sur le site internet du CETMEF dans la base de données CANDHIS

(http://www.cetmef.equipement.gouv.fr)

- en temps différé, sur demande auprès de la DRE Languedoc-Roussillon

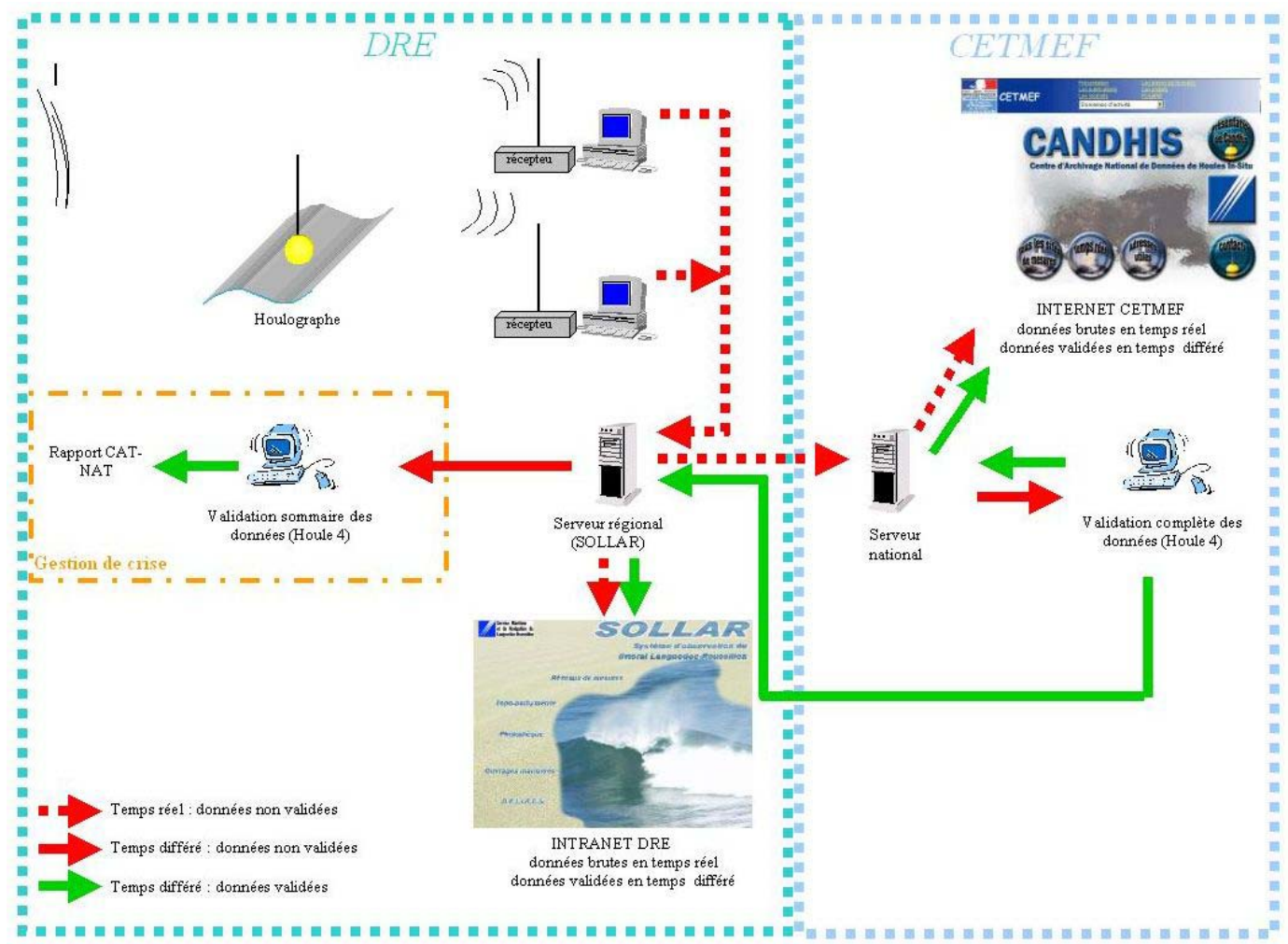

Figure 7. Schéma du transfert des données de houle 


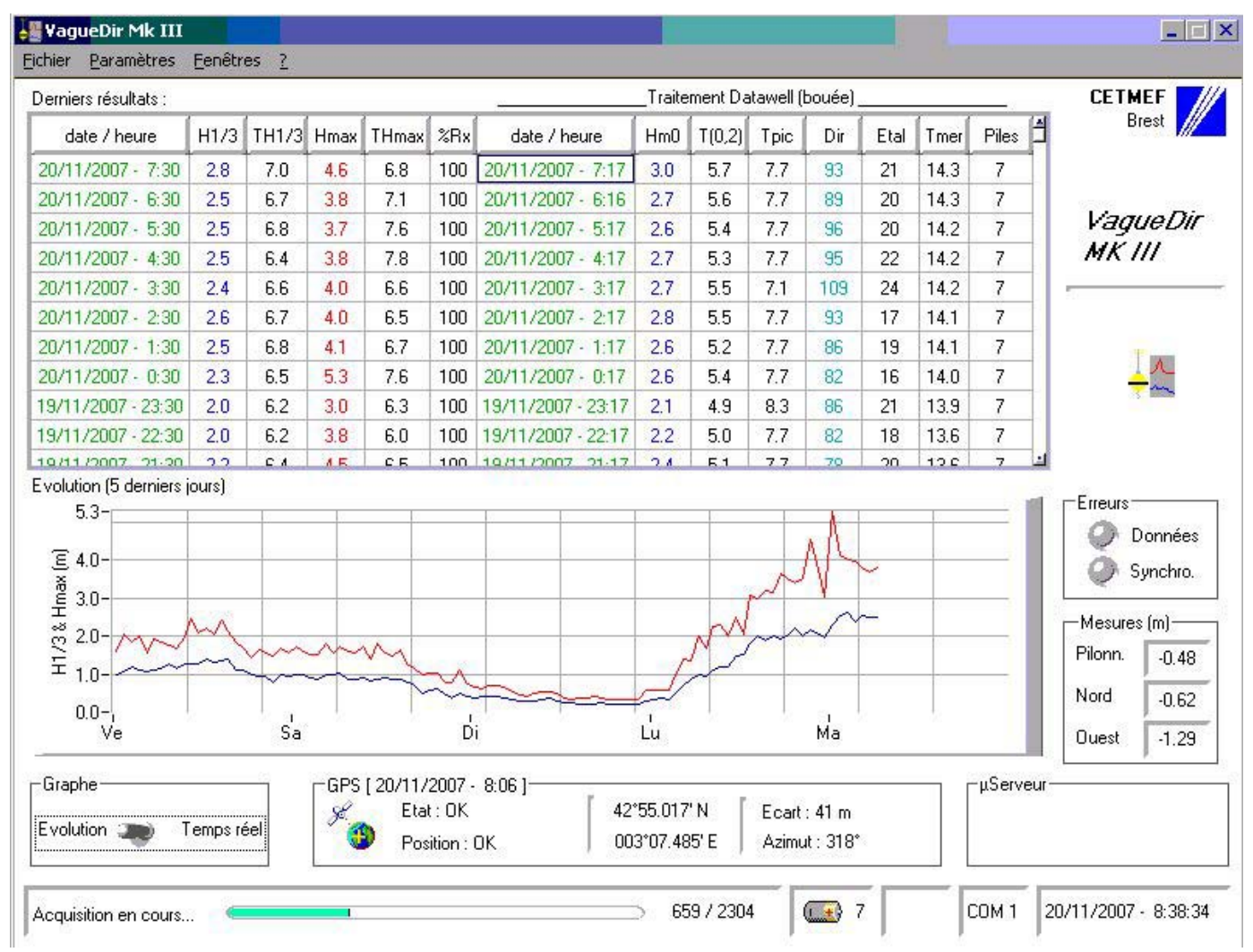

Figure 8. Visualisation des données non validées temps réel sur site de réception

\section{$4 \quad$ Les objectifs de ces mesures:}

Ces mesures présentent de nombreux intérêts parmi lesquels :

- aide à la gestion de crise (temps réel)

- analyse des tempêtes, établissement des rapports de dégâts tempête et des déclarations de catastrophe naturelle (CATNAT)

- amélioration des connaissances et exploitations scientifiques :

- analyse statistique des phénomènes extrêmes

- modélisations hydrodynamiques permettant de mieux appréhender les effets combinés des houles et courants à la côte et ainsi quantifier les déplacements sédimentaires, l'objectif étant la lutte contre l'érosion du littoral. Ces courants proviendront de modèles numériques validés par des campagnes de mesures ponctuelles.

- élaboration de l'atlas des zones inondables par submersion marine et des PPR (Plans de Prévention des Risques)

\section{$5 \quad$ Perspectives}

La DRE envisage de mettre en oeuvre un réseau pérenne de suivis et d'observation des niveaux marins, si possible en temps réel, de marégraphes sur les côtes du Languedoc-Roussillon dont les données seront aussi à disposition des 
SPC (Services des Prévisions des crues) et du SHOM (Service Hydrographique et Océanographique de la Marine).

Concernant le réseau de houlographes, l'installation d'une quatrième bouée directionnelle, au large de Port- Camargue (département du Gard) viendra achever le réseau de mesures et permettra ainsi de couvrir l'intégralité du Golfe de Lion.

\section{$6 \quad$ Conclusion}

Le réchauffement climatique va induire une évolution notable des phénomènes naturels en particulier marins : élévation du niveau de la mer, modification des états de mer (hauteur, période), fréquence des évènements extrêmes ....

Ces réseaux permettront, dans la durée, de mesurer et d'anticiper les conséquences des changements climatiques prévus.

\section{$7 \quad$ Références bibliographiques}

1 CREACH R., (2008). La lettre de RONIM 2008. Rapport, Service Hydrographique et Océanographique de la Marine. URL: http://www.shom.fr/fr_page/fr_act_oceano/maree/lettre_RONIM_2008.pdf 2 GUIZIEN K., (à paraître). Mesures de l'état de mer le long des côtes du Languedoc-Roussillon (1988-2008). Article, Laboratoire d'océanographie biologique Arago à Banyuls/mer

3 HONTARREDE M., JOURDAN R., VAYSSE F., VALANTIN P-Y. (2003). Tempête dans le Golfe du Lion Décembre 2003. Revue MET MAR (Météorologie Maritime), $\mathrm{n}^{\circ} 203$, pp 6-9.

4 SIMON B., (2007). La marée océanique côtière. Coédition de l'Institut Océanographique et du SHOM, $433 \mathrm{p}$.

5 VALANTIN P-Y., (2003). Analyse de la tempête marine du 4 décembre 2003. Rapport, Service Maritime et de Navigation du Languedoc-

Roussillon, $19 \mathrm{p}$.

6 VANROYE C., GARCIA C. (2008). Analyse de la tempête marine sur le littoral Languedoc-Roussillon du 02 au 04 janvier 2008. Rapport, Direction Régionale de l'Equipement du Languedoc-Roussillon, 27 p.

7 VANROYE C., GARCIA C., HEURTEFEUX H. (2007). Analyse de la tempête marine sur le littoral Languedoc-Roussillon du 19 au 22 novembre 2007. Rapport, Direction Régionale de l'Equipement du Languedoc-Roussillon et EID Méditerranée, 27 p. 